\title{
BREVE MEMORIA DE UNA MAESTRA RURAL EN TIEMPOS DE LA EDUCACIÓN GENERAL BÁSICA
}

\author{
Brief memoir of a rural teacher in the times \\ of the General Basic Education
}

Alicia López Pardo ${ }^{\alpha}$

Soy Alicia López Pardo, nacida en una aldea de Lugo, criada en un entrañable seno familiar de nueve miembros, con escasez de recursos materiales, pero con abundancia de alegría y de ciertos valores que dejaron huella en mi vida. Disfruté de una afortunada infancia, en un paisaje de especial belleza, arropada por un próximo y comunitario clima vecinal. Mi etapa de adolescente se desarrolló en buena parte en la ciudad de Lugo, en contacto con un ambiente humanista en el que se fomentan mis inquietudes hacia el mundo rural y el ámbito de la educación.

Intento aportar mis testimonios personales, fundados en mis vivencias durante quince años de coincidencia con la normativa legal de la Ley General de Educación (LGE). Incluyo diversas experiencias profesionales durante el referido marco legal, desarrolladas en las etapas de la Educación General Básica (EGB), en los cuatro primeros de los siete destinos por los que transcurrió mi rodaje profesional. Me sitúo en una amplia etapa vivida con una intensidad de imposible síntesis, por lo que debo limitarme a breves pinceladas sobre algún aspecto de mi trabajo. Por circunstancias que serían largas de detallar, hago dos veces la carrera de Magisterio. La nueva ordenación del sistema educativo con la LGE influyó en la decisión de la segunda carrera, que habilita para especialidades y me permite el acceso a la universidad, circunstancia que me permite hacer las especialidades de Ciencias Sociales y Matemáticas, añadidas al primer título en educación primaria del plan antiguo.

\footnotetext{
^ Nova Escola Galega. Mesa Galega de Educación no Rural. Montecelo, Viñas, 39. Paderne 15319, A Coruña, España. alicialopezpardo@gmail.com
} 


\section{PRIMER DESTINO EN LA ALTA MONTAÑA LUCENSE}

En el año 1975 y recién aprobadas las oposiciones, elijo mi primer destino movida por mi vocación de ejercer en el ámbito rural y en la alta montaña. Sin previo conocimiento del lugar ni de las condiciones, llego a Os Prados, una unitaria situada en una aldea de las montañas de Os Ancares (Cervantes, Lugo), de aquellas consideradas de difícil desempeño, de aspecto tercermundista. Sirva de muestra el estado del edificio escolar, de propiedad privada y que provenía de la precaria rehabilitación de unas cuadras destinadas a vacas, la parte baja, y gallinero la parte alta. En esta última se instaló el aula, a la que se accedía por un corral lleno de barro y excrementos de vacuno, ocupado por reses (vacas, yeguas, sendas paradas de buey y de caballo sementales). Seguía la entrada por una escalera peligrosa y de aspecto ruinoso, igual que la puerta, el piso o las ventanas. Tampoco existían baños, ni luz eléctrica, ni siquiera una estufa en aquella escuela de montaña.

Este primer destino coincide con el confuso momento de una etapa de transición en el sistema educativo. Eran tiempos en los que, en la Galicia rural, se inicia la implantación de aquella reforma educativa en lo que concierne a la planificación y organización escolar. Atravesamos un complejo tránsito; pesan rancios estilos pedagógicos, poderes caciquiles y aún aletea el espíritu franquista. Predominan modelos monolíticos, criterios ajenos a las diversas realidades, carentes de lógica y de una respetuosa adaptación.

Mi llegada a la escola dos Prados causa desconcierto a niños y adultos acostumbrados a un trato distante y sumiso con las maestras. Mi aspecto juvenil provocaba que cualquiera de las alumnas de secundaria podría pasar por maestra, como sucedió en ocasiones en las que, bromeando, jugábamos a confundir a alguien ajeno a la escuela. Al mismo tiempo yo me veo inmersa en unas dificultades que superaban con creces mi imaginación. Me siento en cierto modo turbada frente a la compleja diversidad que percibo en aquel alumnado: en un reducido y deprimente espacio conviven dieciocho alumnos y alumnas de entre cuatro y catorce años (Preescolar y las dos etapas de EBG), cuyo nivel individual real resultaba imposible de perfilar. A simple vista detecto un cúmulo de circunstancias que me desbordan. La mayoría de las niñas, niños y adolescentes para llegar a la escuela caminaban desde aldeas vecinas por 
dificultosos caminos en días de fuertes nevadas. Percibo como aquellas encantadoras criaturas son víctimas de una deficitaria y discontinua atención, siempre condicionada por la provisionalidad y por la falta de asentamiento y de integración de un profesorado flotante, desconectado de aquella realidad. Niños y niñas que arrastran complejo de inferioridad y fuertes bloqueos en su expresión emocional y verbal, principalmente a causa de la represión lingüística sufrida en la escuela por hablar su lengua materna, el idioma gallego. En la misma medida padecían el desprecio por su vida y su cultura. Completaban el panorama aspectos básicos como son las carencias de recursos didácticos, libros de texto inapropiados, faltos de significatividad y desconectado de sus intereses. Todo su contexto escolar constituía un concentrado abono para la desmotivación. En invernales días oscuros, muchas veces a temperaturas bajo cero, con frecuencia era imposible trabajar. Como de tal situación se desentendían la Administración y el Ayuntamiento, después de serios intentos, son las familias quienes aceptan mi propuesta de poner fin al sacrificio soportado por sus hijos e hijas. Madres y padres asumen por su cuenta el arreglo del local y, con una rápida y adecuada obra, se convierte aquel antro en un lindo y hogareño espacio, de lo más acogedor, que incluye una chimenea de leña cuyo fuego directo nos llenaba de magia vital.

Mi compromiso inicial para fomentar esta primera iniciativa comunitaria de las familias será decisivo para afrontar la siguiente fase. De inmediato surge otro gigantesco frente, provocado institucionalmente, al ponerse en marcha el nuevo plan de escolarización, destinado a substituir las escuelas unitarias por las concentraciones escolares. El estereotipo diseñado a nivel estatal resulta ser un despropósito para una realidad tan diferenciada como la alta montaña. Con la nueva ley como pretexto, personas con amplios poderes y completamente ajenas al mundo que pretenden gobernar ponen en marcha un modelo, también importado, de escolarización para la montaña. Se trata del desafortunado sistema de concentración en base a la escuela hogar. Tal régimen suponía el internamiento del alumnado a partir de la tierna edad de cuatro años con la consiguiente separación familiar. Dependiendo de las variables condiciones climatológicas propias de aquella época de fuertes nevadas, la incomunicación total podía durar varias semanas. Una de las primeras escuelas hogar de montaña que se ejecuta es en el municipio de mi 
escuela, Cervantes; por ello es tan directa la afectación que me toca. Yo reacciono inmediatamente para implicarme contra las decisiones amparadas por la Administración a espaldas de la población afectada.

En aquellas aisladas tierras apenas se percibían señales de democracia. Cualquier junta vecinal era perseguida y condenada como mitin no autorizado. El conocimiento del plan implantado llega clandestinamente a las familias, gracias a maestras enteradas y otros agentes solidarios defensores de sus derechos. Surge un activismo con fuerza imparable como negativa a tan inhumano sistema de escolarización. Lo insólito en aquel momento es cómo la oposición al plan oficial se reafirma sobre fundamentos alternativos contundentes. En contrapartida a las llamadas escuelas hogar se activa una respuesta comunitaria que da lugar a la elaboración de un plan de escolarización específico para la montaña. Se consolida dicho proyecto en base a asegurar la proximidad de la escuela, dentro de un correcto enfoque pedagógico, a través de la distinción de sectores geográficos debidamente definidos y de una organización funcional idóneamente planteada. De inmediato se pone en marcha un estudio con la colaboración de personas expertas y nada menos que se diseña un completo plan de escolarización racional, concebido sobre un sistema de pequeñas concentraciones y escuelas mixtas justamente dotadas, con servicio de transporte adecuado para la montaña y demás servicios imprescindibles para los habitantes de aquellas marginadas aldeas. En dicho plan participamos enseñantes, gentes vecinas y personas voluntarias llegadas de diversos lugares, expertas en determinadas cuestiones.

En el año 1978 este proyecto de sorprendente nivel de elaboración se presenta en la Delegación de Educación de Lugo, en el Ministerio de Educación y Ciencia (MEC) y en los medios. Simultáneamente, ante el propio Gobernador Civil de Lugo, se presenta el mismo y se despliega una movilización inaudita en tal época. Cientos de personas se manifiestan contra la falta de receptividad de las autoridades educativas y en protesta por las medidas represivas adoptadas contra personas significadas como opuestas a las decisiones de la Administración. La fuerza del movimiento y la contundencia del proyecto presentado fue rotunda, hasta el punto de conseguir frenar la puesta en funcionamiento de la polémica escuela hogar ya construida con el consiguiente despilfarro presupuestario. De inmediato, mientras se lleva a cabo la realización 
del plan alternativo que había sido reivindicado, se exige la habilitación provisional de locales estratégicamente situados y con las básicas condiciones.

El proyecto alternativo tuvo que ser finalmente reconocido por la Administración. Fue este un hecho pionero tanto en Galicia como en el resto del Estado. Siendo una referencia, progresivamente cunde el ejemplo y se reconoce su potencial para ser adaptado a muchas zonas rurales gallegas y de fuera de Galicia. Se puede afirmar que conserva actualidad para las bases de un plan de educación y desarrollo global específico para muchos ámbitos rurales, tal como seguimos defendiendo hoy en día.

A pesar de sonadas represalias, entre las que se incluirá mi futuro traslado forzoso a Canarias, el logro de esta lucha continúa y llega a ser otra de las referencias pedagógicas históricas. Debo mencionar a aquel llamado grupo de mestras da montaña lucense, con señalado protagonismo en todos estos hechos. Destacamos estas maestras por nuestro trabajo cooperativo, por luchas debidamente cohesionadas y por nuestro compromiso integral en las perspectivas de la Escuela Moderna. La deficiente formación profesional en muchos casos era suplida por un gran empeño autodidacta, por una eficiente investigación-acción sobre la marcha, a través de equipos colaborativos con clara orientación desde la pedagogía Freinet.

Transitamos por una época de avances al retomar aquella pedagogía iniciada por las maestras y maestros republicanos que pagaron con sus vidas su vocación y entrega. Cuantos enseñantes fueron eliminados o represaliados durante la dictadura recuperan presencia sobre todo a través del Movimiento Freinet. Los grupos ACIES (Asociación para la Correspondencia y la Imprenta Escolar) llegan a Galicia y activan su impronta también en las zonas rurales más deprimidas. Vuelve la escuela viva, dinámica, científica, implicada y que no va de innovadora en procura de méritos, sino de renovadora en procura de buenas prácticas.

Aquel clima pedagógico propicia hechos extraordinarios que podemos considerar hasta mágicos, como sucedió en mi propia escuela. ${ }^{1}$ Experiencias como la vivida con una gallina que eligió nuestra escuela para

\footnotetext{
1 Un artículo sobre esta etapa profesional mía, titulado «La magia de la escuela rural», fue publicado en la revista Cuadernos de Pedagogía 325 (2003): 44-49.
} 
poner sus huevos (durante mis tres años de estancia en esa inolvidable escuela, a pitiña se convierte en una compañera que marcará nuestras vidas). Este suceso maravilloso es una experiencia singular, recogida años más tarde en un libro en el que las niñas, los niños y yo contamos nuestra experiencia. ${ }^{2}$ Fue esta una de las ilusionantes motivaciones, compartida mediante la correspondencia escolar, así como en las convivencias y excursiones organizadas entre aquellas interactivas escuelas, entre las que se mantenía una comunicación formidable sin más trazas de Tecnologías de la Información y la Comunicación (TIC) que las imprentas de gelatina, transmitidas por las valiosas técnicas que aportan las prácticas freinetianas. Siempre compartiendo al máximo los escasos recursos fue asombroso como una única maestra o maestro lograba la compaginación de las etapas de EGB, desde párvulos a octavo curso, admitiendo casos que excedían la edad escolar, sumando la preparación para las pruebas de graduado escolar y certificados de escolaridad para alumnado con escasas posibilidades de continuidad. En la mayoría de los casos hay que añadir a lo dicho los atrancos por parte de inspectoras, incluidas amenazas de expediente entre otras, por utilización de la lengua gallega y por prácticas novedosas chocantes en el marco de la escuela tradicional (investigación del entorno, cálculo vivo, periódico escolar y múltiples vivencias que suplantaban a los libros de texto). As mestras da montaña, dentro del amparo del espíritu de la LGE, aunque perseguidas por detractores oficiales, logramos grandes propósitos gracias al apoyo de las familias y de las comunidades entre las que nos consideramos vecinas plenamente incluidas.

\section{SEGUNDO DESTINO: TRASLADO FORZOSO A CANARIAS}

Desde las entrañables montañas de Os Ancares, de las que me sentía enamorada, aquel impensado y forzado destierro provoca un completo vuelco en mi vida personal y profesional. Significa para mí un aterrizaje en las antípodas. Sufrí un duelo por las rupturas inevitables con los apegos a una lucha sin tregua que hervía en retos y logros, a la entrega a criaturas que me provocaban desvelos, a la apasionada aventura con a pitiña... Una ruptura tan drástica supone una prueba de resistencia física y mental. En un estado de desplome, con las fuerzas en límites, apenas podía calibrar

\footnotetext{
2 A historia da pita que elexíu a nosa escola pra poner (Santiago de Compostela: Galaxia, 1986).
} 
los cambios ni las múltiples maravillas y miserias que palpitaban en un mundo tan diferente en el que me tenía que asentar. De una escuela minúscula paso a una de las concentraciones de mayor tamaño y masificación, con sistema de transporte de una complicada red de múltiples líneas de autobuses: Colegio Manuel de Falla en Aguamansa, La Orotava, Tenerife. En aquella época zona demográficamente densa y en la misma desmesura económica y culturalmente deprimida, con acusadas lagunas de marginalidad. En lo que de entrada se presenta como otra realidad, diferenciada e incomparable, según mi integración avanza descubro encubiertos e impensados paralelismos de los que no tenía indicios.

A poco de que me rebelo contra la depresión provocada por aquel injusto y traumático traslado, me sorprendo a mí misma cogiendo aliento para enfrentarme a las primeras luchas en defensa de los derechos de determinados alumnos. Nada más llegar a mi forzoso destino afronto un fuerte incidente en defensa de unos adolescentes considerados problemáticos sobre los que pesaba la amenaza de ser expulsados del colegio o expedientados por problemas de adaptación y disciplina. Como consecuencia de mi atrevimiento a oponerme a tan injusta y disparatada medida tuve que asumir la desmesurada responsabilidad de hacerme cargo de ellos. Ante situaciones inverosímiles que no se pueden resumir, me enfrento a la dirección y a la inspección después de ser amenazada por negarme a hacer una programación de oficio, para nada adaptada al nivel ni a los intereses de aquel alumnado víctima del sistema social y escolar. Exijo mi derecho, amparada por la LGE, a realizar una programación coherente, comprometida y motivadora, aproximada a las necesidades y posibilidades de aquellos muchachos, igual que exijo un tiempo para yo integrarme mínimamente en su mundo y conocer sus carencias y expectativas. La reacción provocada fue motivo de la primera amenaza de expediente que recibí en aquel destino, pero la empatía que me inspiraban mis alumnos me dio la suficiente fuerza para mantenerme en mis propósitos. Fue una difícil y arriesgada decisión de la que llegué a estar muy satisfecha y gracias a ella llegaron a presenciar cambios sorprendentes hasta quienes intentaron reducirme profesionalmente por salirme de lo usualmente establecido y por desacato a la autoridad.

En el segundo año me toca asumir la dirección de aquel gigantesco colegio, con cincuenta docentes en el claustro más varias aulas externas adscritas al mismo. Algunas, ubicadas en los barrios más distantes 
y vulnerables de la zona alta de la Orotava y camino de las Cañadas del Teide, eran aulas habilitadas en condiciones de vergonzosa precariedad. Me veo en la obligación de aceptar la dirección, pero pongo como condición una organización peculiar de dirección colegiada, ya experimentada en algún otro caso referencial. Esto llevó a un compromiso de la mayoría del claustro, que asume el plan de trabajo cooperativo que propuse entre mis condiciones. En escaso tiempo el cambio se plasmó en una visible transformación tanto en la marcha pedagógica como en la gestión del colegio. También dio su fruto en el ambiente renovador mi contacto con el colectivo de pedagogía Freinet de Tenerife, que a mi llegada a la isla se encontraba en período de gestación, y mi relación con la Universidad de la Laguna, en la que cursé estudios con compañeras afines.

Conmigo se traslada a latitudes canarias mi rodaje en las montañas gallegas de Os Ancares. A pocos meses de asentarme en la isla voy descubriendo como el mismo fenómeno, con distintas apariencias, se repite en todas partes. Economía contra pedagogía, planificación irracional contra ajuste a las necesidades, masificación contra humanización, macrotransporte contra proximidad... Me sirvió totalmente el modelo defendido en Galicia -todos los criterios encajaban- para enfrentarme a la reclamación de un plan de descentralización del macrocolegio Manuel de Falla. Era evidente la necesidad de una reestructuración en pro de la creación de pequeñas agrupaciones y/o pequeñas escuelas mixtas, debidamente dotadas y distribuidas con equidad por el territorio rural. Se requirió un proceso exigente, pero se dieron avances en buen sentido.

Esta y otras incontables batallas y denuncias ante la opinión pública por el estado calamitoso de las instalaciones, del comedor, del transporte y de otros servicios me arriesgaron a dos nuevas amenazas de expediente, por la responsabilidad que mi cargo de directora requería. Tales amenazas fueron frenadas con contundencia por la fuerza ejercida desde un claustro unido y plenamente comprometido y una Asociación de padres y madres de alumnos (APA) de centenares de familias creada sobre la lucha común. La consecución de lo que se reclamaba fue un hecho mostrado en breve tiempo, así como la visible transformación del colegio resultó notable y duradera.

A mi regreso de Tenerife vine acompañada de las más sentidas lágrimas de despedida y de vínculos personales con gentes con quienes, 
plenamente integrada, compartí de corazón inquietudes, luchas y enorme disfrute. Este mi segundo destino permanece en mis memorias y se prolonga activamente y con actualidad en otros proyectos vitales.

\section{TERCER DESTINO: COLEGIO LUIS SANTÁNGEL (EL SALER, VALENCIA)}

En la década de los ochenta, cuando me incorporo a este destino, era este colegio conocido por su considerable prestigio. Hay que añadir que tanto su emplazamiento, entre un hermoso pinar al lado de la playa, como sus óptimas instalaciones convertían al colegio de El Saler en un centro idóneo. Dicho reconocimiento estaba fundado en una excelente orientación pedagógica, unida a un gran nivel de coordinación y de trabajo cooperativo, sumada a la vez la participación intensa de una activa APA.

Me tocó desarrollar mi trabajo docente en la segunda etapa de EGB, dentro de un contexto desconocido para mí y con diferencias tan acusadas respecto a Canarias como encontré entre los anteriores destinos. De entrada, tuve la gran ventaja de contar con muy buena acogida y con un esmerado apoyo por parte del equipo directivo y de todo el claustro. Gocé además de la afortunada coincidencia de colaborar con una compañera extraordinaria, Josua Mijares,$^{3}$ con formación y experiencia admirable en la entonces llamada educación especial inclusiva.

Este destino supuso para mí una oportunidad especial de formación continua y de profundización en la línea avanzada del enfoque esencial de la LGE. También me aportó la ocasión de contacto y apertura a la diversidad cultural y en especial a la lengua del País Valencià. Compaginando con el trabajo escolar, también aproveché otros contactos mediante mis estudios en la Facultad de Psicología de Universidad de Valencia. En su conjunto mi paso por El Saler resultó para mí una experiencia enriquecedora y satisfactoria.

\footnotetext{
3 Josua Mijares Cibrián, integradora de múltiples aspectos como desarrollo global, psicomotricidad, grafoterapia y musicoterapia. Destacada fue su labor en formación de adultos y más tarde también reconocida pionera en animación terapéutica con criaturas en situación límite en el hospital oncológico La Fe de Valencia.
} 


\section{CUARTO DESTINO, VUELTA A GALICIA: COLEXIO DE EXB DE PONTECARREIRA (FRADES, A CORUÑA)}

Cuando no me lo esperaba y como había sido mi destierro, de manera tan anómala que no podría explicar en breve, se produce mi traslado a Galicia. Regreso a un lugar para mí desconocido y no deseado, lejos de mi provincia natal. Me depara el destino una deprimente sorpresa, la antítesis de la experiencia anterior: una enorme concentración de pésimo diseño y peores instalaciones, masificada a tope y con una gestión lamentable en su conjunto. La dirección me adjudica un aula de Preescolar, la única etapa en la que yo carecía de especialidad y de experiencia. Me encuentro con un reducido espacio en un aula con 32 alumnos, mezclados, de 4 y 5 años. Eran la mayoría niñas y niños que se incorporaban ese año. Casi todos sufrían un penoso transporte, comían en el comedor y muchos pasaban fuera de su casa desde las 8 a las 18 horas, una situación originada por el ya cuestionado plan de escolarización implantado en el ámbito rural gallego. Durante aquella inapropiada generalización de la reforma derivada de la LGE, fue denominador común el cierre de las escuelas unitarias y el incalificable transporte a un centro masificador.

Me resultó aleccionadora mi primera experiencia en Galicia en un centro de estas características, convertido en cuartel de una infancia mal tratada y desarraigada de su ambiente familiar. En general, el sistema de largos desplazamientos del alumnado y del profesorado implicaban el sacrificio de los primeros y la falta de integración y de compromiso de los segundos casi siempre desconectados de las familias y del medio natural del alumnado. Los materiales imperantes eran productos de un extendido negocio de las editoriales. En contra de lo que pretendía fomentar el espíritu de la LGE, mediante una operativa red de distribuidores las coproducciones uniformadoras para todo el estado español frenaban la globalización activa, la vivencia e investigación del propio medio, la creatividad y todo tipo de recursos naturales.

Mi reacción ante el desalentador panorama fue la convencida insumisión. Desde el primer momento busco alianzas con algunos equipos cooperativos y grupos activos que surgen vigorosos en la época. De nuevo me embarco en la acción comprometida, en la misma orientación 
iniciada con las mestras da montaña lucense y con el colectivo Freinet de Canarias. De nuevo, en defensa de viejas causas, me veo involucrada en enfrentamientos peliagudos con la Administración. Continuos ataques y amenazas, que darían para un grueso volumen, fueron cotidianos durante los cinco primeros años regidos por el mismo director al amparo de una inspectora de su mismo perfil. Después de un cambio de dirección, logrado a pulso a base de unir fuerzas con una APA implicada en máximos, se alcanzan nuevas miras y mejoras considerables.

Mi opción pedagógica se consolida con mi primera promoción, formada por un alumnado con el que desde preescolar hasta $5^{\circ}$ de EGB experimento una continuidad de siete años y con el que me impliqué de lleno profesional y emocionalmente. Perduran hasta la actualidad mutuos vínculos establecidos con aquellas niñas y niños. En aquel referido clima de acoso a mi persona y mis métodos (hasta el colmo de una intervención de la propia Guardia Civil que actúa ilegalmente manipulada con intención de atemorizar), fue decisivo el apoyo de sus familias a mi línea pedagógica. Me resulta inexpresable mi agradecimiento y admiración hacía aquellas madres y padres que, en todo momento y con valentía inusual en aquel contexto, valoraron en mi trabajo y actuaron en defensa de mis métodos pedagógicos y de mi estilo de escuela. Fue tal su implicación que llegó a desautorizar severas amenazas de expediente promovidas desde la mencionada dirección del centro.

Después de Pontecarreira, ya transcurrida la época de la LGE, paso por otros lugares hasta el momento de mi jubilación. Durante los años destinada en el programa Preescolar na Casa me especializo en Educación Infantil, más tarde concurso al Colexio de Educación Infantil e Primaria (CEIP) de Pontedeume, siendo mi último destino en la Escola de Vixoi-centro rural agrupado de Bergondo, A Coruña.

Expreso mi agradecimiento por el legado que recibí, en tiempos de la EGB y posteriores, por parte de docentes históricos, maestras y maestros freinetianos, así como por el empuje de un largo camino de trabajo cooperativo disfrutado con compañeras y compañeros inolvidables que siguen alentando mis pasos. 


\section{REFERENCIAS}

López Pardo, Alicia. «La magia de la escuela rural». Cuadernos de Pedagogía 325 (2003): 44-49.

López Pardo, Alicia. A historia da pita que elexíu a nosa escola pra poner. Santiago de Compostela: Galaxia, 1986. 